\title{
Análisis epidemiológico de la depresión perinatal
}

\author{
Epidemiological analysis of perinatal depression
}

\author{
Nilson A. Contreras-Carreto ${ }^{1}$, Luis Villalobos-Gallegos ${ }^{2}$ y Javier Mancilla-Ramírez ${ }^{3 *}$ \\ ${ }^{1}$ Dirección de Mejora de Procesos, Dirección General de Calidad y Educación en Salud (DGES), Secretaría de Salud, Ciudad de México; ${ }^{2}$ Facultad \\ de Medicina y Psicología, Universidad Autónoma de Baja California, Campus Tijuana, Tijuana, Baja California; ${ }^{3}$ Sección de Estudios de Posgrado \\ e Investigación, Escuela Superior de Medicina, Instituto Politécnico Nacional, Ciudad de México. México
}

\section{Resumen}

La depresión perinatal es un problema habitualmente subdiagnosticado que no sólo afecta de manera importante la salud mental y física materna, sino también al recién nacido en los aspectos físico, mental y cognitivo, con serias repercusiones en la vida adulta. A pesar de los esfuerzos para obtener información útil para dimensionar el problema, la mayoría de los expertos en el tema coinciden en señalar que este trastorno es más frecuente de lo que se estima. La mejora en la obtención de información en salud mental perinatal permitirá dimensionar mejor el problema y una adecuada toma de decisiones en salud mental pública para disminuir la morbimortalidad y la carga de la enfermedad asociada a la depresión perinatal.

Palabras clave: Depresión perinatal. Trastornos mentales perinatales. Salud mental maternal.

\begin{abstract}
Perinatal depression is a commonly underdiagnosed problem which not only severely affects maternal mental and physical health, but also affects the newborn on a physical, mental and cognitive level with serious repercussions on adult life. Despite efforts to obtain useful information to dimension the problem, most experts in the field agree that this disorder is more frequent than estimated. Thus, the improvement in obtaining information on perinatal mental health will not only allow for a better dimensioning of the problem, but will also allow for better decision making in public mental health to reduce morbidity and mortality and the burden of disease associated with perinatal depression.
\end{abstract}

Keywords: Perinatal depression. Perinatal mental disorders. Maternal mental health.

\section{Introducción}

El embarazo es para la mujer el periodo de máxima vulnerabilidad, por lo que se requieren cuidados integrales, ya que estos repercuten en el desenlace del embarazo y en la salud futura del recién nacido'. El diagnóstico de depresión perinatal frecuentemente es desestimado por los profesionales de la salud y pocas veces es tomado en cuenta en la planeación de políticas en salud'. Por ello, realizar una revisión sustancial de la evidencia epidemiológica existente sobre depresión perinatal puede sensibilizar en el tema.

\section{Correspondencia:}

*Javier Mancilla-Ramírez

Marina Nacional, 60 


\section{Definición de depresión perinatal}

La depresión perinatal es un problema de salud pública que es necesario atender para disminuir la morbimortalidad materna y perinatal asociada a este padecimiento ${ }^{2}$. Se encuentran algunas referencias ya en los escritos de Hipócrates (469-337 a.C.), de Joao Rodrigues de Castelo Branco (1511-1568) y de Luis de Mercado (1531-1611) ${ }^{3-6}$. Durante los siglos XVI$\mathrm{XVII}$, la depresión perinatal se asoció a filicidio ${ }^{3,7}, \mathrm{y}$ para principios del siglo $\mathrm{XX}$ ya se diferenciaba claramente si los síntomas depresivos se presentaban antes, durante o posterior al embarazo.

Durante el siglo $\mathrm{XX}$, la tristeza, la psicosis y la melancolía se consideraban parte de la depresión posparto. Sin embargo, hoy se consideran afecciones diferentes y forman parte del diagnóstico diferencial ${ }^{8}$. A finales del siglo $X X$, con el desarrollo de la salud mental como campo de estudio, fue necesario recolectar datos estadísticos para homogenizar las definiciones de los trastornos. Así, en 1952 se publicó el Diagnostic and Statistical Manual of Mental Disorders (DSM) de la American Psychiatric Association, como complemento de la sexta edición de la Clasificación Internacional de Enfermedades (CIE-6), la cual corresponde a la versión en español de la International Statistical Classification of Diseases and Related Health Problems (ICD) publicada por la Organización Mundial de la Salud y que tuvo su origen en la Lista de causas de muerte de 1893.

En cuanto a los trastornos depresivos, el DSM-IV TR no hacía una clara definición de la depresión perinatal y se utilizaban los mismos criterios para definir el trastorno depresivo mayor (TDM) de la población general. EI TDM presentó muy pocos cambios con respecto al DSM-5, el cual lo define según los siguientes criterios:

- En el transcurso de 2 semanas deben presentarse al menos cinco de los siguientes síntomas casi todos los días: aumento o pérdida de peso, insomnio o hipersomnia, agitación o retraso psicomotor, fatiga o pérdida de energía, sentimientos de inutilidad o culpa, disminución de la capacidad para la toma de decisiones y pensamientos recurrentes de muerte o ideación suici$\mathrm{da}$, de los cuales al menos uno debe acompañarse de un estado de ánimo deprimido o pérdida de interés o de placer.

- La sintomatología debe causar un deterioro importante y significativo en el funcionamiento social, laboral o de otras áreas del individuo.
- El episodio no se puede atribuir a efectos fisiológicos de alguna sustancia u otra afección médica ${ }^{9-12}$.

Aún en la actualidad, la depresión perinatal se describe con diferentes nombres, dependiendo del momento en que se presenta: depresión prenatal y depresión posparto. Sin embargo, el término «depresión perinatal» hace referencia a ambos periodos. La depresión perinatal se define como la ocurrencia de un TDM durante el embarazo o después del nacimiento 0 de la adopción de un bebé13. Hasta el DSM-5 se describió como «TDM con inicio en el periparto», haciendo énfasis en que el $50 \%$ de los trastornos depresivos posnatales en realidad tienen su inicio durante el periodo antenatal, por lo que utilizar el término «periparto» sería lo más adecuado. Por ello, el diagnóstico debe ser considerado desde el inicio del embarazo y hasta 1 año posterior al parto ${ }^{9,13}$.

En cuanto a la CIE-10, la depresión perinatal se clasifica en dos grandes categorías:

- Como parte de los trastornos mentales y del comportamiento («trastornos mentales y del comportamiento asociados con el puerperio, no clasificados en otra parte»).

- Como parte de las enfermedades que complican el embarazo, el parto y el puerperio ("otras enfermedades maternas clasificadas en otra parte, pero que complican el embarazo, el parto y el puerperio») $)^{14}$.

\section{Distribución poblacional de la depresión perinatal}

La depresión perinatal es multifactorial y afecta cualquier tipo de parto y número de gestación ${ }^{11}$. Las estadísticas son heterogéneas y dependen del país, la institución y el autor que las publica. Ello puede deberse a la diversidad de criterios utilizados, al momento y la ventana de tiempo que evalúan, al subdiagnóstico o a una falta de sensibilización y capacitación de los profesionales de la salud y de educación para la salud en la población general. En todo el mundo, la prevalencia de depresión antenatal va del $7-15 \%$ en los países con altos ingresos económicos al $19-25 \%$ en aquellos con bajos a medianos ingresos económicos ${ }^{15}$. En cuanto a la depresión posnatal, su prevalencia en los países con altos ingresos es del $10-15 \%$, de aproximadamente el $20 \%$ en los de bajos a medianos ingresos y tan alta como del 30\% en los países asiáticos ${ }^{15-17}$. En México, se estima que un $30.7 \%$ de las mujeres sufren depresión en algún 
momento del embarazo y hasta 1 año después del parto ${ }^{18,19}$. Habitualmente, la sintomatología se inicia a las 6-12 semanas posparto y tiene una duración de 7 meses $^{8,20}$. Hasta en el $50 \%$ de estas mujeres se identifica algún trastorno del sueño $0^{20,21}$.

Langan y Goodberg ${ }^{22}$ identificaron los siguientes factores de riesgo: antecedente de depresión (odds ratio [OR]: 29.0), miedo al parto (OR: 3.8), tabaquismo (OR: 3.25 ), adolescencia (OR: 3.14), ser madre soltera (OR: 2.86), bajo nivel socioeconómico (OR: 2.59), edad $\geq 40$ años (OR: 1.41), violencia doméstica (OR: 3.1), ansiedad materna (OR: 2.7), embarazo no deseado (OR: 1.41) y diabetes gestacional (OR: 2.29). Por su parte, Blom et $\mathrm{al}^{23}$ identificaron otros factores de riesgo: preeclampsia (OR: 2.58), hospitalización durante el embarazo (OR: 2.25), cesárea de emergencia (OR: 1.53) y complicaciones neonatales (OR: 1.56). $Y$ en mujeres mexicanas se han identificado, además de los anteriores: educación secundaria (OR: 5.61), desempleo (OR: 3.57), abortos (OR: 2.56), bajo apoyo social (OR: 5.76), eventos vitales estresantes (OR: 3.95), depresión prenatal (OR: 2.84), baja resiliencia (OR: 3.05$)^{24}$; violencia (OR: 3.9$)^{25}$; recién nacido mujer (OR: 2.38 ), miedo al parto (OR: 2.11) ${ }^{26}$; estrés prenatal (OR: 5.80 ), estrés durante el parto (OR: 7.71), mala relación de pareja (OR: 7.03) ${ }^{27}$; y número de embarazos (OR: 2.3) ${ }^{28}$.

Rochat et al. ${ }^{29}$ describieron como sintomatología depresiva prevalente: estado de ánimo deprimido o pérdida del interés o del placer (21.10\%), estado de ánimo deprimido más pérdida del interés o del placer $(50.46 \%)$, cambios en el apetito o en el peso corporal (97.5\%), fatiga $(63.3 \%)$, cambios en la capacidad de concentrarse $(54.13 \%)$, trastornos del sueño $(53.21 \%)$, culpa excesiva o inapropiada $(53.21 \%)$, agitación o retraso psicomotor (41.28\%) e ideación suicida (27.52\%).

Los primeros 3 meses posparto son el periodo de mayor vulnerabilidad en la mujer ${ }^{30}$, por lo que se recomienda atención en los siguientes síntomas de alarma: quejas psicosomáticas, cansancio, dolores diversos, solicitud repetida de ayuda, tristeza y preocupación constante, o miedo ${ }^{31,32}$.

\section{Carga de enfermedad por depresión perinatal}

En la actualidad, no existen métricas específicas para la depresión perinatal, pues estas se incluyen en las correspondientes a la población general. En 1990, los trastornos depresivos fueron la cuarta causa de discapacidad en el mundo, ascendiendo al tercer lugar en $2017^{33}$. En el año 2000 se reportaron 6582 muertes secundarias a trastornos depresivos. En cuanto a las métricas comparadas de 1990 con respecto a 2000, se observó un incremento en la estimación de años vividos con discapacidad del 10.7\% al $12.1 \%$, además de un incremento en los años de vida ajustados por discapacidad del $3.7 \%$ al $4.46 \%$. Del mismo modo, existen diferencias epidemiológicas por regiones, pues la carga por trastornos depresivos en África es tan baja como el $1.2 \%$ y en América Latina tan alta como del $8.9 \%$. En los países con bajos a medianos ingresos económicos, los años de vida ajustados por discapacidad son del $4.1 \%^{34}$.

La depresión perinatal produce un costo social elevado por los gastos en salud que representa y por el desempleo, el ausentismo laboral y la pérdida de productividad global que conlleva. Para la Organización para la Cooperación y el Desarrollo Económicos, el gasto en salud por trastornos mentales puede llegar hasta el $4 \%$ del producto interior bruto nacional. El retraso en su atención puede llevar al infanticidio o al suicidio, siendo esta la principal causa de muerte materna durante el primer año posparto ${ }^{15,35}$. Otros factores que pueden incrementar los gastos en salud son el aborto espontáneo, la prematuridad, la preeclampsia, las complicaciones obstétricas, el bajo peso al nacer y las complicaciones neonatales ${ }^{36-39}$. El TDM frecuentemente afecta el vínculo madre-hijo ${ }^{37}$. También, los hijos de madres deprimidas tienen mayor riesgo de desarrollar problemas emocionales 0 trastornos mentales en la infancia y la vida adulta ${ }^{12,40,41}$.

\section{Determinantes sociales y depresión perinatal}

Los determinantes de la salud mental favorecen o afectan de manera directa o indirecta la salud mental materna ${ }^{42}$. Por ello, el medio ambiente adverso, la contaminación, la pobreza, la malnutrición, el consumo de drogas, el estrés o el excesivo trabajo físico pueden afectar negativamente la salud mental y física de la mujer y del feto ${ }^{43}$. Fisher et $a l .^{44}$ identificaron como factores protectores la educación superior (OR: 0.5), tener una pareja con empleo (OR: 0.3) y los cuidados posparto por un familiar o persona de confianza (OR: 0.4). Dentro de los determinantes sociales se identificaron el bajo acceso a los servicios de salud, los bajos ingresos y las dificultades financieras (OR: 2.1), pertenecer a una minoría étnica o religiosa (OR: 2.1), la adicción en la pareja (OR: 1.96), 
la violencia intrafamiliar (OR: 1.1), la unión polígama (OR: 7.7), el insuficiente apoyo social o familiar (OR: 2.8) y vivir en un área rural (OR: 2.1$)^{44}$. En la literatura nacional se describen, además, la minimización de la sintomatología por la paciente, la familia o el personal de salud, el abandono de la pareja por separación o divorcio, la falta de redes de apoyo, el antecedente de abuso físico, emocional o sexual, la mala relación familiary la dificultad para el cuidado de otros hijos ${ }^{31,44-46}$.

\section{Estrategias de prevención}

Para disminuir la morbimortalidad por depresión perinatal es fundamental ${ }^{31}$ :

- Brindar información suficiente, clara y precisa a la mujer, su pareja y familia.

- Control de la sintomatología.

- Disminuir el miedo y la angustia.

- Fortalecer el vínculo madre-hijo y con la pareja.

- Promover el buen funcionamiento familiar.

- Intervenir en los estresores actuales.

- Fortalecer las redes de apoyo familiares, sociales e institucionales.

Se han descrito buenos resultados con las siguientes estrategias: creación de áreas especializadas en salud mental perinatal (OR: 0.30$)^{47}$, visitas domiciliarias (OR: 0.71), seguimiento telefónico (OR: 0.36), coaching interactivo (OR: 0.41), psicoterapia individual y grupal cognitivo-conductual (OR: 0.51), intervenciones psicoeducativas madre-hijo (OR: 0.47$)$ y realización de actividad física (OR: 0.49) 2,22,48.

En México, a pesar de que se cuenta con la guía Prevención, diagnóstico y manejo de la depresión prenatal y posparto en el primero y segundo niveles de atención (2014), ésta no especifica los mecanismos para su implementación y, desafortunadamente, su utilidad para la planeación de recursos y la implementación de las estrategias necesarias es limitada. Hasta el momento, la única estrategia global es la educación en todos los niveles ${ }^{18}$.

\section{Conclusiones}

En México, la prevalencia de depresión perinatal sólo es conocida por las investigaciones realizadas por diversos autores, principalmente en instituciones de salud mental, y la mayoría de los hospitales gineco-obstétricos no cuentan con áreas para la atención de la salud mental perinatal. Por lo tanto, el problema podría ser más grave en comparación con lo que se publica $^{49}$. Los estudios reflejan métricas limitadas y poco representativas de la población general. Para mejorar el flujo de información es necesario eficientar el sistema de referencia-contrarreferencia entre instituciones que brindan atención obstétrica e instituciones especializadas en salud mental, además de la optimización del Sistema Nacional de Vigilancia Epidemiológica para la creación de estrategias y acciones que permitan producir información epidemiológica útil para la salud mental pública.

Uno de los primeros pasos que podría considerarse es la inclusión de sistemas activos de detección de depresión perinatal, en particular en unidades maternofetales, neonatales y de consulta externa obstétrica. Se recomienda el uso de instrumentos de tamizaje validados previamente, como la Edinburgh Postnatal Depression Scale ${ }^{50}$. Así, no sólo se identificaría oportunamente el trastorno mental, sino que, al anexarse el resultado en los expedientes clínicos, mejorarían las estadísticas hospitalarias.

\section{Financiamiento}

Este manuscrito no recibió financiamiento público ni privado.

\section{Conflicto de intereses}

Los autores declaran no tener conflicto de intereses.

\section{Responsabilidades éticas}

Protección de personas y animales. Los autores declaran que para esta investigación no se han realizado experimentos en seres humanos ni en animales.

Confidencialidad de los datos. Los autores declaran que en este artículo no aparecen datos de pacientes.

Derecho a la privacidad y consentimiento informado. Los autores declaran que en este artículo no aparecen datos de pacientes.

\section{Bibliografía}

1. Marcos R, Rodríguez M, Izquierdo N, Olivares M, Soto C. Depresión perinatal: rentabilidad y expectativas de la intervención preventiva. Clínica y Salud. 2019;28:49-52.

2. Stewart D, Robertson E, Dennis CL, Grace SL, Wallington T. Postpartum depression: literature review of risk factors and interventions. Toronto: University Health Network; 2003.

3. Maldonado J. Salud mental perinatal. Washington, DC: Organización Panamericana de la Salud; 2011.

4. Ramón F, Mansilla A, Rivera A. Neurofisiología para estudiantes de medicina. Facultad de Medicina, UNAM. (Consultado el 12-09-2020.) Disponible en: http://www.facmed.unam.mx/Libro-NeuroFisio 
5. Kokkinaki T. Maternal and paternal postpartum depression: effects on early infant-parent interactions. J Preg Child Health. 2015;2:e126.

6. Rojo A. Biografía del Doctor Luis de Mercado. Spanish Journal of Surgical Research. 2015;18:189-97.

7. De la Espriella R. Filicidio: una revisión. Rev Colomb Psiquiatr. 2006;35:71-84

8. Thurgood S, Avery D, Williamson L. Postpartum depression (PPD). Am J Clin Med. 2009;6:17-22.

9. American Psychiatric Association. Manual Diagnóstico y Estadístico de los Trastornos Mentales DSM-5. Ciudad de México: Panamericana; 2014.

10. Kupfer D. Depression and the new DSM- 5 classification. Medicographia. 2014;36:521-5.

11. Demyttenaere K. Affect modulation, functioning, and depression. Medicographia. 2014;34:441-5

12. Rafferty J, Mattson G, Earls M, Yogman M. Incorporating recognition and management of perinatal depression into pediatric practice. Pediatrics. 2019;143:e20183260.

13. Centro Nacional de Excelencia Tecnológica en Salud (CENETEC); 2014 (Consultado el 20-09-2020.) Disponible en: https://cutt.ly/Mg2PFJi

14. World Health Organization (WHO). International Classification of Diseases $11^{\text {th }}$ Revision. (Consultado el 22-09-2020.) Disponible en: https://icd who.int/browse11

15. Gelaye B, Rondon MB, Araya R, Williams MA. Epidemiology of maternal depression, risk factors, and child outcomes in low-income and middle-income countries. Lancet Psychiatry. 2016;3:973-82.

16. Pearson R, Carnegie R, Cree C, Rollings C, Rena-Jones L, Evans J, et al. Prevalence of prenatal depression symptoms among 2 generations of pregnant mothers: the Avon Longitudinal Study of Parents and Children. JAMA Network Open. 2018;1:e180725.

17. Sikander S, Ahmad I, Bates L, Gallis J, Hagaman A, O'Donnell K. Cohort profile: perinatal depression and child socioemotional development; the Bachpan cohort study from rural Pakistan. BMJ Open. 2019;9:e025644.

18. Lara MAL, Patiño $P$, Navarrete L, Nieto L. Depresión posparto, un problema de salud pública que requiere de mayor atención en México. Género y Salud en Cifras. 2017; 15:12-22.

19. Anokye R, Acheampong E, Budu-Ainooson A, Obeng El, Akwasi AG. Prevalence of postpartum depression and interventions utilized for its management. Ann Gen Psychiatry. 2018;17:1-8.

20. Flores M. La salud mental en la mujer embarazada. Perinatol Reprod Hum. 2013;27:143-4.

21. Chan E, Cisneros C, Martín S, Reyes A. El insomnio como factor de riesgo para la depresión en mujeres embarazadas. Perinatol Reprod Hum. 2013;27:171-6.

22. Langan R, Goodbred A. Identification and management of peripartum depression. Am Fam Physician. 2016;93:852-8.

23. Blom $E$, Jansen $P$, Verhulst $F$, Hofman $A$, Raat $H$, Jaddoe $V$, et al. Perinatal complications increase the risk of postpartum depression. The Generation R Study. BJOG. 2010;117:1390-8.

24. Lara M, Navarro C, Navarrete L, Cabrera A, Almanza J, Morales F. Síntomas depresivos en el embarazo y factores asociados, en pacientes de tres instituciones de salud de la Ciudad de México. Salud Mental. 2006;29:55-62.

25. DeCastro F, Hinojosa N, Hernández B. Risk and protective factors as sociated with postnatal depression in Mexican adolescents. J Psychosom Obstet Gynaecol. 2011;32:210-7.

26. DeCastro F, Place J, Billings D, Rivera L, Frongillo EA. Risk profiles associated with postnatal depressive symptoms among women in a public sector hospital in Mexico: the role of sociodemographic and psychosocial factors. Arch Womens Ment Health. 2015;18:463-71.

27. Alvarado C, Sifuentes A, Estrada S, Salas C, Hernández B, Ortiz S. Prevalencia de depresión posnatal en mujeres atendidas en hospitales públicos de Durango. Gac Med Mex. 2010;146:1-9.

28. Almanza J, Salas C, Olivares A. Prevalencia de depresión posparto y factores asociados en pacientes puérperas de la Clínica de Especialidades de la Mujer. Rev Sanid Milit Mex. 2011;65:78-86.
29. Rochat TJ, Tomlinson M, Bärnighausen T, Newell ML, Stein A. The prevalence and clinical presentation of antenatal depression in rural South Africa. J Affect Disord. 2011;135:362-73.

30. Mendoza C, Saldivia S. Actualización en depresión postparto: el desafío permanente de optimizar su detección y abordaje. Rev Med Chile. 2015;143:887-94.

31. Medina E. Diferencias entre la depresión postparto, psicosis postparto y la tristeza postparto. Perinatol Reprod Hum. 2013;27:185-93.

32. Caparros R. Depresión postparto: un problema de salud pública mundial. Rev Panam Salud Publica. 2018;42:e97.

33. Institute for Health Metrics and Evaluation. Findings from the Global Burden of Disease Study 2017. Seattle: IHME; 2018.

34. Ustun T, Ayuso-Mateos J, Chatterji S, Matters C, Murray J. Global burden of depressive disorders in the year 2000. Br J Psychiatry. 2004;184:386-92.

35. Carreño S, Medina ME. Panorama epidemiológico de los trastornos mentales, su impacto entre el balance trabajo-familia. 2018. (Consultado el 15-09-2020.) Disponible en: https://cutt.ly/Kg2PAQA

36. Maldonado M, Lartigue T. Cambios fisológicos y emocionales durante el embarazo normal y la conducta del feto. En: Maldonado $\mathrm{M}$, Brockington I, editores. La salud mental y sus dificultades en la etapa perinatal. Washington, DC: Organización Panamericana de la Salud; 2011.

37. Jadresic E. Depresión perinatal: detección y tratamiento. Rev Med Clin Condes. 2014;25:1019-28.

38. Bitew T, Hanlon C, Kebede E, Honikman S, Fekadu A. Antenatal depressive symptoms and perinatal complications: a prospective study in rural Ethiopia. BMC Psychiatry. 2017;17:301.

39. Lartigue T, Maldonado JM, González BI, Sauceda JM. Depresión en la etapa perinatal. Perinatol Reprod Hum. 2008;22:111-31.

40. De Vicente A, Castilla C, Villamarín S, Verdulllas S. La maternidad: mucho más que una revolución emocional. Infocop. 2016;73:4-5.

41. Lasheras G, Farré B, Gelabert E, Ferraz L, Mestre G, Rovira I. Noticias de salud mental perinatal. C Med Psicosom. 2015;113:51-8.

42. Ministerio de Salud de Costa Rica. Política Nacional de Salud Mental 2012-2021. San José: Gobierno de Costa Rica; 2012.

43. Ministerio de Salud de Chile. Modelo de Gestión Centro de Salud Mental Comunitaria. Santiago: Gobierno de Chile; 2018.

44. Fisher J, Cabral M, Patel V, Atif P, Tran T, Holton S. Prevalence and determinants of common perinatal mental disorders in women in low- and lower-middle-income countries: a systematic review. Bull World Health Organ. 2012;90:139G-49G

45. Maldonado M, Sauceda J. Fenómenos de "programación" in utero: alto nivel de estrés psicosocial y desnutrición durante el embarazo. En: Maldonado M, Brockington I, editores. La salud mental y sus dificultades en la etapa perinatal. Washington, DC: Organización Panamericana de la Salud; 2011.

46. Satyanarayana V, Lukose A, Srinivasan K. Maternal mental health in pregnancy and child behavior. Indian J Psychiatry. 2011;53:351-61.

47. US Preventive Services Task Force. Interventions to prevent perinatal depression: US Preventive Services Task Force recommendation statement. JAMA. 2019;321:580-7.

48. O'Connor E, Senger C, Henninger M, Coppola E, Gaynes B. Interventions to prevent perinatal depression: evidence report and systematic review for the US Preventive Services Task Force. JAMA. 2019:321:588-601.

49. Constanza M, Sandra S. Actualización en depresión postparto: el desafío permanente de optimizar su detección y abordaje. Rev Med Chile. 2015; 143:887-94

50. Earls MF, Yogman M, Mattson G, Rafferty J, Committee on Psychosocial Aspects of Child and Family Health. Incorporating recognition and management of perinatal and postpartum depression into pediatric practice. Pediatrics. 2010;126:1032-9. 